\title{
RESEARCH
}

Open Access

\section{Quality of life data for individuals affected by spinal muscular atrophy: a baseline dataset from the Cure SMA Community Update Survey}

\author{
Lisa Belter ${ }^{*} \mathbb{D}$, Rosángel Cruz and Jill Jarecki
}

\begin{abstract}
Background: Individuals and/or caregivers of individuals affected by spinal muscular atrophy (SMA) completed the 2019 Cure SMA Community Update Survey, online, assessing health-related quality of life (HRQoL), loss of work productivity, and fatigue using the Health Utilities Index Questionnaire (HUI), the Work Productivity and Activity Impairment Questionnaire (WPAl), and the Patient Reported Outcomes Measurement Information System Fatigue Short Form (PROMIS Fatigue SF), respectively. The purpose was to collect baseline quality of life results among individuals affected by SMA using the above Patient Reported Outcome Measures (PROMs).
\end{abstract}

Results: Of 666 surveys completed between March and May 2019, 478 were included in this analysis, accounting for duplicates, missing data, or deaths. The breakdown across SMA type I, II and III was 25, 47 and 28\%, respectively. Responses were characterized by current functional status/milestone, with subsets for "permanent ventilation," "non-sitters," "sitters," "walk with support," and "walk alone." WPAI and HUI respondents included affected adults and caregivers. The PROMIS Fatigue SF was completed by the primary caregiver of affected children.

Overall, those affected by a less severe form of SMA and with a higher functional status reported higher HRQOL and lower work productivity and activity impairment. All affected individuals reported higher fatigue levels than the general population.

Conclusions: This study offers useful insights into the burden of SMA among affected individuals and their caregivers. The results provide a baseline picture of the patient and caregiver experience with SMA in a posttreatment era from which to measure year-over-year changes in quality of life scores from new therapies and improved care. The WPAI demonstrates the significant impact of work productivity among SMA populations. Aspects of the HUI seem more appropriate to certain SMA sub-populations than others. Measures from the PROMIS Fatigue SF appear to under-represent the burden of fatigue often reported by SMA individuals and caregivers; this may, perhaps be due to a lack of sensitivity in the questions associated with fatigue in the SMA affected population, when compared with other studies on this topic. Overall, these results suggest the need for SMAspecific quality of life outcome measures to fully capture clinically meaningful change in the SMA population.

Keywords: Spinal muscular atrophy, Health utilities index, Work productivity and activity impairment, PROMIS fatigue short form, Patient reported outcome measures, Health related quality of life

\footnotetext{
*Correspondence: lisa.belter@curesma.org

Cure SMA, Elk Grove Village, IL, USA
}

(c) The Author(s). 2020 Open Access This article is licensed under a Creative Commons Attribution 4.0 International License, which permits use, sharing, adaptation, distribution and reproduction in any medium or format, as long as you give appropriate credit to the original author(s) and the source, provide a link to the Creative Commons licence, and indicate if changes were made. The images or other third party material in this article are included in the article's Creative Commons licence, unless indicated otherwise in a credit line to the material. If material is not included in the article's Creative Commons licence and your intended use is not permitted by statutory regulation or exceeds the permitted use, you will need to obtain permission directly from the copyright holder. To view a copy of this licence, visit http://creativecommons.org/licenses/by/4.0/ The Creative Commons Public Domain Dedication waiver (http://creativecommons.org/publicdomain/zero/1.0/) applies to the data made available in this article, unless otherwise stated in a credit line to the data. 


\section{Background}

Spinal muscular atrophy (SMA) is a genetic neuromuscular disease characterized by progressive muscle atrophy and weakness that historically often led to paralysis and premature death [1]. While SMA most often first presents among infants and toddlers, it also can present in juveniles and, less frequently in adults. SMA has traditionally been classified into clinical subtypes based upon age of onset and the highest physical milestone achieved [2].

Type I SMA (Werdnig Hoffman Disease) presents with severe generalized weakness, hypotonia, tongue fasciculations, and impaired respiratory function, difficulty feeding and swallowing, among other symptoms appearing within the first 6 months of life. In the absence of treatment, most of these infants required intensive supportive care, as they were never able to sit, and lost their ability to breathe and feed independently. Historically, more than half of children with type I SMA did not survive to age two [3]. This is changing with new FDA approved therapies [4, 5]. Type II SMA (Kugelberg Welander disease) usually presents between the ages of 6 and 18 months, with affected children being able to sit independently but unable to walk without assistance, with most experiencing respiratory difficulties and progressive scoliosis, and some developing eventual problems with chewing and swallowing. Patients with juvenile SMA (type III) can walk at some point and have a normal life expectancy, although they develop muscle weakness over time and often eventually lose ambulation. In rare cases, patients' symptoms first appear in adulthood (type IV) [6-8].

As mentioned, the SMA treatment landscape changed dramatically in late 2016 with FDA approval of nusinersen, the first disease modifying therapy for SMA, and then with the FDA approval of onasemnogene abeparvovec-xioi in 2019, demonstrating that it is possible to impact rates of survival, motor function, and respiratory function for SMA patients $[5,9,10]$. Access to these novel therapies coupled with improvements in supportive care are not only rapidly changing the therapeutic landscape but also the quality of life of those affected and how they may experience SMA [5, 10, 11]. Despite this progress, there is limited qualitative and quantitative data that have been collected on the burden of SMA, particularly after the approval of these treatments.

What we have learned through qualitative research conducted prior to approval of new treatment options, is that the burden of SMA is multifaceted and extremely challenging for patients and their families. Often this odyssey begins with a prolonged and traumatic process to confirm diagnosis and a life-long journey of overwhelming physical, emotional, psychosocial, and financial strains associated with managing and living with a progressive, debilitating, and incurable disease [7, 12-15].

As we continue to capture and optimize care and treatment access in SMA, it is critical to quantify outcomes that are meaningful from the patient perspective, and which measure the impact of therapies on other dimensions of life other than assessing survival or significant changes in motor milestones. For instance, the Institute for Clinical and Economic Review (ICER) published a report, in April 2019, on the FDA-approved treatments called Spinraza ${ }^{\oplus}$ and Zolgensma ${ }^{\oplus}$ for Spinal Muscular Atrophy: Effectiveness and Value [16]. The original ICER report found that neither medication met the standard quality-adjusted life year (QALY) costeffectiveness threshold nor did they meet the modified QALY threshold for rare diseases, even though both drugs are transformative when given early.

Importantly, it has also been suggested by the patients themselves, as well as healthcare providers, that the factors being assessed in such pharmacoeconomic analyses are not sensitive enough to capture the overall impact on quality of life [17]. Thus, it has been proposed for SMA (and for rare disease more generally) that focusing on generating evidence that translates therapy benefit from clinical trials to patient and family relevant outcomes such as quality of life, independence and productivity impact might be more appropriate when analyzing cost effectiveness. Encompassing these additional dimensions would provide a more complete picture of the burden of disease and the potential overall impact of a new therapy [18]. With similar efforts recently undertaken in other rare, pediatric diseases such as Duchenne Muscular Dystrophy (DMD), there has been strong interest in collecting health utility and HRQoL measures for the SMA population [19].

This study looks at various PROMs that assess specific aspects of the patient experience that might be more sensitive in capturing subtle, but meaningful changes with drug therapy. Leveraging its annual online Community Update Survey [20], in 2019 Cure SMA engaged respondents in completing three commonly used PROMs: the Health Utilities Index (HUI), Work Productivity and Activity Impairment (WPAI) and the Patient Reported Outcomes Measurement Information System Fatigue Short Form (PROMIS Fatigue SF).

The study assesses various PROMs that address specific aspects of the patient experience to determine whether the PROMs are sensitive in capturing subtle, but meaningful changes with drug therapy administration in order to benchmark the progression of SMA, and other aspects of disease impact such as quality of life, work productivity, and fatigue. The authors also present a discussion of the strengths and limitations of these 
three PROM tools when used in the SMA setting, suggesting the need for future efforts to develop and validate SMA-specific outcome measures.

\section{Methods \\ Participants}

The Cure SMA database members were the primary target population for these surveys. Cure SMA is the largest SMA patient advocacy organization based in the US and maintains the largest self-reported database on individuals with SMA worldwide [21]. More specifically, the patient-reported database contains self-reported records for more than 8000 affected individuals, including information relating to demographics, SMA type, and diagnosis date [21]. Beginning in 2017, the organization launched an annual online Community Update Survey of this database to more comprehensively capture the natural history of SMA, from the patient's perspective and to develop additional data that can support assessment of the impact of SMA. In the 2019 survey participants were asked to complete information on a range of topics including demographic information (e.g., sex, age at survey, vital status, educational level, employment); clinical disease characteristics (e.g., age at diagnosis and symptom onset, SMN2 gene copy number) and family history; respiratory interventions, motor function, surgeries and hospitalizations; and participation in clinical trials and treatment. Additionally, the survey included a specific assessment of HRQoL, work productivity and activity impairment, and fatigue. To minimize survey fatigue among a chronically affected patient community, only three assessments covering HRQoL, work productivity and activity impairment were used in the 2019 survey. Details on a previously used assessment in the 2017 Community Update Survey, the PedsQL ${ }^{\mathrm{mm}}$ [22], was presented at the 22nd International Congress of the World Muscle Society [23]. Respondents were stratified within the final dataset by the affected individual's SMA type and current functional status. Functional status was categorized as requiring permanent ventilation (defined as 16 or more hours per day of breathing support); nonsitters (defined as lacking head control, voluntary grasping, voluntary kicking or roll-over completely and not requiring permanent ventilation); sitters (defined as able to sit without support, stand alone or stand with assistance); walk with support; and walk independently. These states were treated as mutually exclusive and identified from the survey responses.

Over 4000 survey invitations were sent out to both parents of affected children and affected adults within the Cure SMA database in March 2019 via email and mailed postcards. It was also posted to the Cure SMA website and social media sites. Surveys were accepted from both US and international families. IRB approval was obtained by Western IRB (IRB Report ID: 1785926). All data were de-identified prior to analysis.

\section{Outcome measures \& statistical approach Health utilities index}

To assess overall health-related quality of life utility scores within the SMA population, Cure SMA used the Health Utilities Index Mark 3 (HUI3) system as its tool [24]. It defines 972,000 unique health states. This classification system provides a comprehensive framework within which to describe health status and develop assessment of health-related quality of life (HRQL) and has been used successfully in rare disease states impacting children [19] ages 5 and up, but has not yet been validated in an SMA population. Health-related quality of life (HRQoL), has been defined as "the value assigned to duration of life as modified by the impairments, functional states, perceptions, and social opportunities that are influenced by disease, injury, treatment, or policy [25]." Although there are other systems available within the HUI, such as the HUI Mark 1 (HUI1) and the HUI Mark 2 (HUI2) only the HUI3 results are included in this analysis. The HUI1 was originally developed to assess the health of children who had been in neonatal intensive care and is now seldom used [24]. The HUI2 assesses seven attributes of quality of life: vision, hearing, speech, mobility, emotion, cognition, self-care, pain and fertility. The HUI3 assess eight attributes of quality of life: vision, hearing, speech, ambulation, dexterity, emotion, cognition and pain. Both the HUI2 and HUI3 results were computed following the survey, but given the HUI3 provided more discrimination across functional status than the HUI2 and to prevent redundancy of results, Cure SMA concluded that only the HUI3 data will be presented here as it provided the more detailed and relevant descriptive system, full structural independence and population norms most relevant to SMA. This additional baseline data for the SMA population can be used in secondary analyses and future comparative studies.

For this analysis, a single global utility score was developed for sub-types based on functional milestone to isolate health quality of life experience among the relevant sub-populations. HUI scores are calculated using the HUI3 health status classification system and utility scoring function. HUI scores range from -0.36 (worst possible health state) through 0.00 (death) to 1.00 (perfect health). Alternative to the continuous HUI3 utility scores, is to group them into disability categories, such that a score of 1.00 represents perfect health, scores of 0.89-0.99 represent mild disability, scores of 0.70-0.88 represent moderate disability, and scores less than 0.70 represent severe disability [26]. Additionally, the scores of the eight single attributes of quality of life as defined by the HUI3 health status classification system (vision, 
hearing, speech, ambulation, dexterity, emotion, cognition, and pain) are also presented here by SMA type. The scores range from 0.00 (death) to 1.00 (perfect health) [24]. Due to the non-normal distribution of the attribute scores by SMA type, the Kruskal-Wallis $\mathrm{H}$ test was used to determine statistically significant differences between attribute scores by type. The HUI was completed by parents of affected children ages 5 and up and affected adults ages 18 and up. Children did not complete the HUI.

\section{Work productivity and activity impairment questionnaire}

Cure SMA selected the Work Productivity and Activity Impairment Questionnaire (WPAI) [27] to assess impact of SMA on productivity for both affected adults and caregivers of affected children. The WPAI is a sixquestion survey assessing the effect of a problem on an individual's ability to work and perform regular activities with the prior 7 days. The WPAI has been validated for use in a variety of disease states and has been used in SMA clinical trials [28] but not validated in an SMA population. The assessment tool yields scores on work time missed (absenteeism), impaired productivity at work (presenteeism), overall work productivity loss (absenteeism and presenteeism combined) and impairment in non-work-related activities due to health problems (activity impairment). WPAI outcomes are expressed as impairment percentages, with higher numbers indicating greater impairment and less productivity. Independent ttests were used to test for statistically significant differences in work productivity scores of a caregiver verses an affected adult to determine if work productivity was impacted differently among those caring for someone with SMA versus someone affected with SMA. Where noted, as required Mann-Whitney $U$ tests were used for testing differences in work productivity scores with nonnormal distributions. A linear regression was used to determine if work productivity was impacted by the age of the affected individual. A linear regression was chosen because both the independent variables (WPAI subscores) and dependent variable (age during survey) were continuous variables.

\section{PROMIS fatigue SF}

Fatigue is a common concern among patients with SMA [29]. Fatigue can be described by two domains: an objective one, also referred to as fatigability (susceptibility to decline in motor performance due to weakness or loss of strength) and a subjective one, also referred to as perceived fatigue (which consists of the sense of tiredness or lack of energy) [30,31]. While fatigability is commonly evaluated during clinical assessments, perceived fatigue has only begun to be studied in the SMA population. To assess perceived fatigue, Cure SMA selected the
PROMIS Fatigue SF parent proxy survey instrument [32] because of its available parent proxy version, limited set of questions and free public access. This tool measures the experience and impact of fatigue among various populations but has not yet been validated in an SMA population. Fatigue is measured over the prior 7 days using a 10-item assessment tool. Higher scores indicate greater fatigue. For the SMA survey, the form was completed by parents of affected children between the ages of 5 and 17. For this analysis, T-scores were developed to rescale the raw score into a standardized score with a mean of 50 and a standard deviation of 10 . Scoring was stratified by SMA type and functional milestone and compared to the general US population. Due to the small sample sizes of each subpopulation, statistical significance testing was not done. However, to explore further which question(s) among the PROMIS Fatigue SF may not be sensitive or appropriate to use in the SMA population, a separate factor analysis was carried out using a one-way ANOVA statistical test to assess the relationship between each question of the PROMIS Fatigue SF by SMA type and functional status.

\section{Results \\ Demographics}

In total, 666 responses representing 639 unique individuals were received between March and May 2019, with the majority, 481, filled out on behalf of the affected individual by a parent/caregiver and 185 filled out directly by an affected individual (18 years of age and older) (Fig. 1).

Thirty-four duplicate surveys completed for the same affected individual (i.e.: both parents submitted surveys), 63 surveys missing functional status information, 20 surveys relating to deceased individuals, 55 surveys missing diagnosis date and 16 surveys with SMA type other than I, II, or III were excluded from the final dataset. A total of 478 records, 121 representing type I (25\%), 225 representing type II (47\%) and 132 representing type III (28\%), remained, but not every assessment was completed by all survey participants because of age and type of respondent. The final dataset included 194 affected males and 284 affected females, with an average age of just over 17 years. Survey respondents had a mean age at symptom onset of 2.6, 10.7, and 33.9 months for types I, II, and III respectively, and had a mean age of diagnosis at 4.0, 24.6, and 110.4 months (Table 1).

Among those representing type I affected individuals, $32.23 \%$ required permanent ventilation, $26.45 \%$ were non-sitters but did not require permanent ventilation, $38.84 \%$ were sitters, and $2.48 \%$ could walk with support. Among those representing type II affected individuals, $4.44 \%$ required permanent ventilation, $28 \%$ were nonsitters, $56 \%$ were sitters, $7.56 \%$ could walk with support 
Table 1 Demographics of Affected Individual within Final Dataset for 2019 Community Survey Quality of Life Analysis

\begin{tabular}{|c|c|c|c|c|}
\hline & Total & Type I & Type II & Type III \\
\hline n (\%) & 478 & $121(25.3)$ & $225(47.1)$ & $132(27.6)$ \\
\hline Male, n (\%) & $194(40.6)$ & $54(44.6)$ & $86(38.2)$ & $54(40.9)$ \\
\hline Age at symptom onset in months, mean (SD) & $15.1(31.3)$ & $2.6(2.4)$ & $10.7(5.8)$ & $33.9(54.1)$ \\
\hline Diagnostic delay in months ${ }^{a}$, mean (SD) & $26.9(67.9)$ & $2.1(2.3)$ & $11.9(30.3)$ & $74.4(108.5)$ \\
\hline Age at diagnosis in months, mean (SD) & $43.1(83.2)$ & $4.0(4.4)$ & $24.6(39.5)$ & $110.4(126.2$ \\
\hline Age at time of survey in years, mean (SD) & $17.1(16.8)$ & $4.1(5.9)$ & $17.2(14.3)$ & $28.6(18.8)$ \\
\hline
\end{tabular}

Data represents unique individuals-only

SD standard deviation

${ }^{\mathrm{a}}$ Diagnostic delay calculated for each individual by age at diagnosis (in months) minus age at symptom onset (in months)

and $4 \%$ could walk independently. Among those with type III SMA, $8.33 \%$ were non-sitters, $36.36 \%$ were sitters, $15.91 \%$ could walk with support and $39.39 \%$ could walk independently (Fig. 2). It is important to note that some of these atypical motor functions for their corresponding SMA type may have been due to response error, clinical trial participation and/or receiving a commercially approved therapy. That analysis is not shown here.

\section{HUI3}

Overall, the average HUI3 scores ranged from -0.05 to 0.64 (Table 2). When looking at functional status with the HUI data, we observed that as functional status increased, the health utility rating also increased. For example, the average HUI3 score was 0.24 among those whose maximum motor function was sitting, and it increased to 0.64 for independent walkers. The mean HUI3 scores increased by increasing functional status and SMA type, with the lowest average HUI3 score among those with type I on permanent ventilation, 0.05 , and the highest average HUI3 score of 0.64 among those with type III that can walk independently. All scores regardless of SMA type and functional status remained in the severe disability category (less than 0.70) [26].

When examining the eight individual single-attribute HUI3 utility scores by SMA type, vision, hearing, and emotion (Table 3) had high utility scores (all above 0.9) across all three SMA types, representing none to mild disability. Additionally, there was no statistically significant differences in their utility scores across type. Ambulation was the attribute with the lowest utility scores, $0.01,0.04$, and 0.32 , for type I, II, and III, respectively, representing severe disability, and there was a statistically significant difference in scores by type. The speech attribute utility score was 0.51 in SMA type I, representing severe disability, but the other SMA types scores, 0.95 and 0.99 , for type II and type III, respectively, represented mild to severe disability. Additionally there, was a statistically significant difference by type among the speech attribute.

\section{WPAI}

For the WPAI, sub-scores were stratified by caregiver and affected adults, and by functional milestone of individuals with SMA as reflected in the final dataset (Table 4). Results from the WPAI indicated that 47.95 and $22.01 \%$ of affected adults and caregivers,

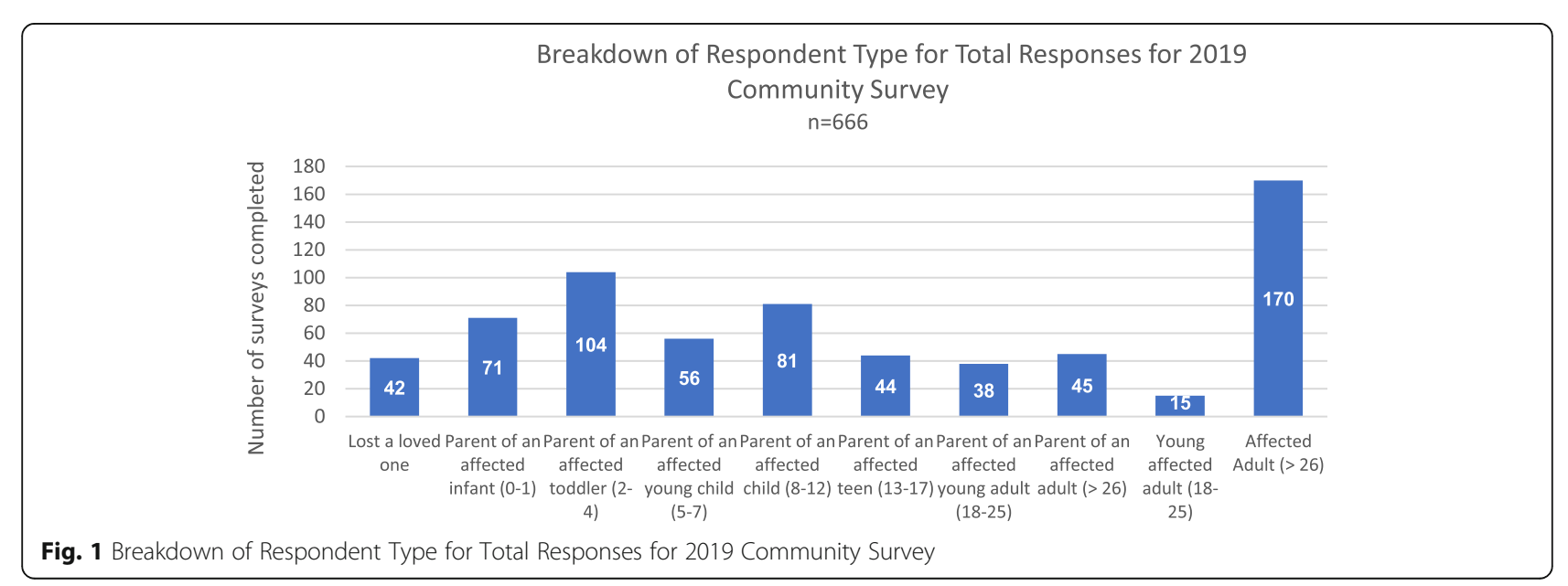


Table 2 HUI3 Scores by Functional Milestone and SMA Type

\begin{tabular}{|c|c|c|c|}
\hline Functional Status & Type I & Type II & Type III \\
\hline \multicolumn{4}{|c|}{ Permanent Ventilation } \\
\hline $\mathrm{n}$ & 18 & 9 & 0 \\
\hline Mean Score (SD) & $-0.05(0.10)$ & $0.10(0.11)$ & \\
\hline Range & $(-0.20-0.13)$ & $(-0.02-0.31)$ & \\
\hline \multicolumn{4}{|l|}{ Non-Sitters } \\
\hline$n$ & 5 & 51 & 9 \\
\hline Mean Score (SD) & $0.06(0.10)$ & $0.12(0.12)$ & $0.14(0.13)$ \\
\hline Range & $(-0.03-0.21)$ & $(-0.16-0.41)$ & $(-0.10-0.34)$ \\
\hline \multicolumn{4}{|l|}{ Sitters } \\
\hline$n$ & 4 & 81 & 41 \\
\hline Mean Score (SD) & $0.11(0.21)$ & $0.26(0.16)$ & $0.23(0.11)$ \\
\hline Range & $(-0.14-0.35)$ & $(-0.20-0.70)$ & $(0.04-0.48)$ \\
\hline \multicolumn{4}{|l|}{ Walk with Support } \\
\hline$n$ & 0 & 6 & 13 \\
\hline Mean Score (SD) & & $0.44(0.12)$ & $0.35(0.21)$ \\
\hline Range & & $(0.32-0.59)$ & $(0.02-0.63)$ \\
\hline \multicolumn{4}{|l|}{ Walk Independently } \\
\hline$n$ & 0 & 3 & 42 \\
\hline Mean Score (SD) & & $0.58(0.15)$ & $0.64(0.24)$ \\
\hline Range & & $(0.48-0.76)$ & $(-0.04-1)$ \\
\hline
\end{tabular}

respectively, were currently employed (working for pay) at the time of the survey. Results on absenteeism, presenteeism, and overall impairment among affected individuals on permanent ventilation were not reported here due to small sample size $(n<2)$. There was a statistically significant difference in the percentage of work missed among caregivers of a child with SMA that could sit and an affected adult that could sit, with caregivers reporting higher percentage of work missed in the last 7 days. Additionally, there was a statistically significant difference in productivity lost in non-work related activities among caregivers of affected children that could walk independently and affected adults that could walk independently with higher productivity lost reported among the affected adults.

Among caregivers, the greatest levels of activity impairment were experienced among those caring for affected individuals on permanent ventilation, 83.1\%). While caregivers of affected individuals that can walk independently have the lowest levels of productivity loss, as one would expect, across all the functional milestone groups, all caregivers are still experiencing a $25.5 \%$ loss of overall work productivity (overall impairment) and $41.2 \%$ loss of non-work activity impairment.

A linear regression analysis (Table 5) was completed to determine if work productivity was impacted by the age of the affected individual. Absenteeism was inversely related to the age of the affected individual, with increasing levels of absenteeism with decreasing age of the affected individual. Age of the affected individual did not explain variance in presenteeism, overall impairment and activity impairment.

\section{PROMIS Fatigue SF}

For assessment of fatigue, parents of affected children ages 5-17 completed the PROMIS Fatigue SF assessment tool and results were stratified by functional milestone and by SMA type. Higher scores on this measurement indicate greater fatigue. Raw scores were rescaled to standardized T-scores to allow for relevant comparison among sub-groups and to the overall US population (Fig. 3).

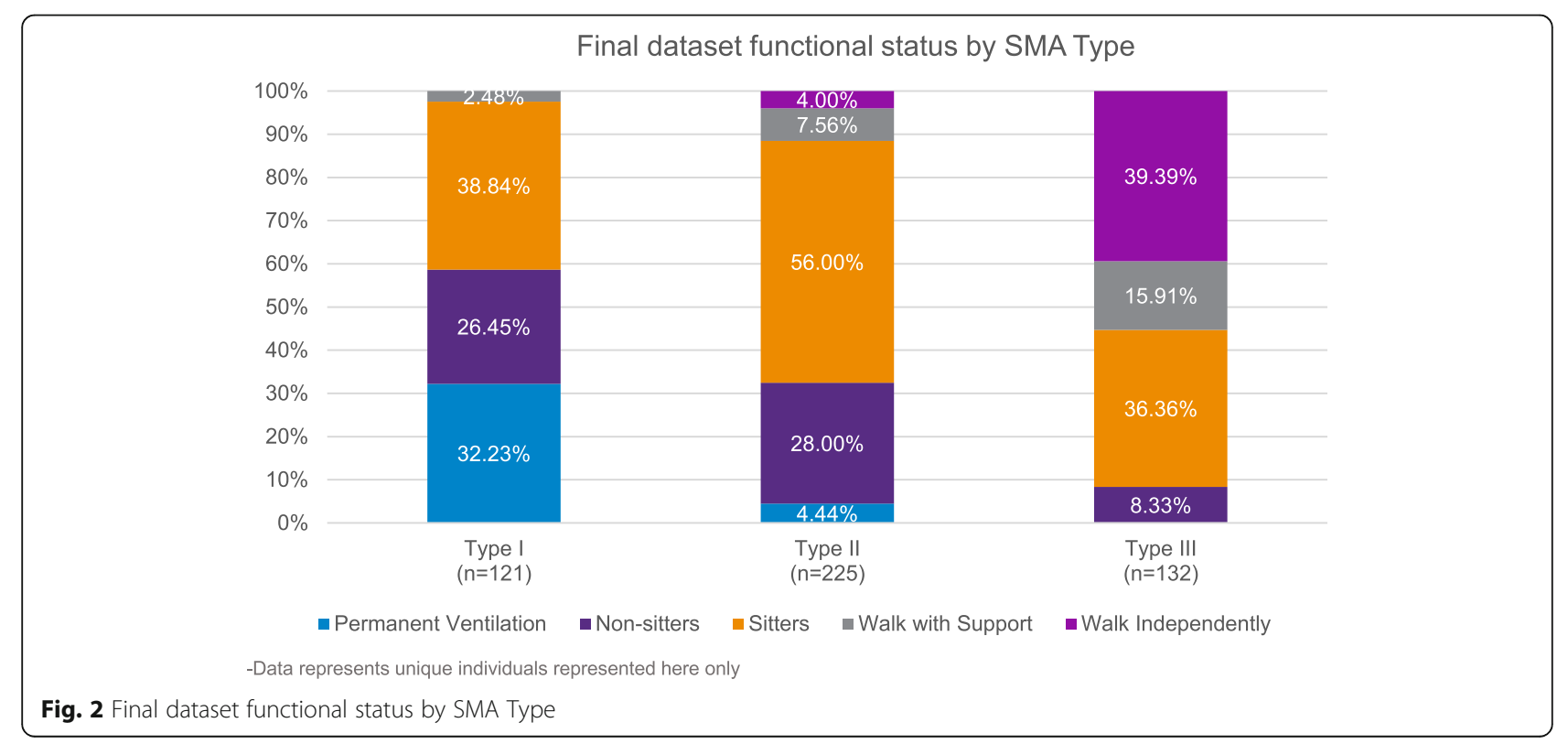


Table 3 HUI3 Attribute Scores by SMA Type

\begin{tabular}{|c|c|c|c|c|}
\hline Attribute Scores & Type I & Type II & Type III & $p$ value \\
\hline \multicolumn{5}{|l|}{ Vision } \\
\hline $\mathrm{n}$ & 29 & 170 & 116 & 0.54 \\
\hline Mean Score (SD) & $0.97(0.05)$ & $0.97(0.06)$ & $0.97(0.03)$ & \\
\hline Range & $(0.73-1)$ & $(0.59-1)$ & $(0.95-1)$ & \\
\hline \multicolumn{5}{|l|}{ Hearing } \\
\hline$n$ & 30 & 167 & 118 & 0.60 \\
\hline Mean Score (SD) & $0.99(0.05)$ & 1.0 & $0.99(0.05)$ & \\
\hline Range & $(0.71-1)$ & $(0.48-1)$ & $(0.48-1)$ & \\
\hline \multicolumn{5}{|l|}{ Speech } \\
\hline $\mathrm{n}$ & 31 & 168 & 118 & $0.0001^{*}$ \\
\hline Mean Score (SD) & $0.51(0.43)$ & $0.95(0.11)$ & $0.99(0.04)$ & \\
\hline Range & $(0-1)$ & $(0.41-1)$ & $(0.67-1)$ & \\
\hline \multicolumn{5}{|l|}{ Ambulation } \\
\hline $\mathrm{n}$ & 31 & 171 & 122 & $0.0001^{*}$ \\
\hline Mean Score (SD) & $0.01(0.06)$ & $0.04(0.14)$ & $0.32(0.36)$ & \\
\hline Range & $(0-0.36)$ & $(0-1)$ & $(0-1)$ & \\
\hline \multicolumn{5}{|l|}{ Dexterity } \\
\hline $\mathrm{n}$ & 31 & 168 & 120 & $0.0001^{*}$ \\
\hline Mean Score (SD) & $0.13(0.27)$ & $0.57(0.35)$ & $0.78(0.27)$ & \\
\hline Range & $(0-1)$ & $(0-1)$ & $(0-1)$ & \\
\hline \multicolumn{5}{|l|}{ Emotion } \\
\hline $\mathrm{n}$ & 30 & 166 & 120 & 0.10 \\
\hline Mean Score (SD) & $0.94(0.14)$ & $0.96(0.08)$ & $0.93(0.13)$ & \\
\hline Range & $(0.33-1)$ & $(0.33-1)$ & $(0.33-1)$ & \\
\hline \multicolumn{5}{|l|}{ Cognition } \\
\hline$n$ & 30 & 167 & 118 & $0.001^{*}$ \\
\hline Mean Score (SD) & $0.87(0.25)$ & $0.97(0.09)$ & $0.96(0.11)$ & \\
\hline Range & $(0-1)$ & $(0.32-1)$ & $(0.32-1)$ & \\
\hline \multicolumn{5}{|l|}{ Pain } \\
\hline$n$ & 31 & 169 & 120 & 0.09 \\
\hline Mean Score (SD) & $0.90(0.11)$ & $0.86(0.16)$ & $0.84(0.16)$ & \\
\hline Range & $(0.48-1)$ & $(0-1)$ & $(0-1)$ & \\
\hline
\end{tabular}

*Statistically significant at $p<0.05$

The adjusted $\mathrm{T}$-scores ranged from 55.69-57.61 when assessing PROMIS scores by functional status. All the scores were worse than the average general population score of 50, but there did not appear to be a trend of scores increasing or decreasing by SMA type or functional status (Table 6).

A factor analysis evaluated the results from each question to determine if there was a statistically significant difference between the average value of the answer and the independent categories of either SMA type or the functional status (Sup. Table 1).

For example, in question 1, the average response answer was 2.71 for type I, 2.34 for type II, and 2.78 for type III and there was no statistically significant difference between these averages. More specifically, the question elements "My child felt weak" and "My child was so tired it was hard for him/her to pay attention" showed a statistically significant (or nearly statistically significant) relationship to SMA type and functional status. However, weakness is a hallmark characteristic of SMA, so these questions may not be reflective of the fatigue an affected individual typically experiences. The other question elements, such as "My child was too tired to do sports or exercise" and "Being tired make it hard for my child to play or go out with friends as much as he/she would like" did not have any statistical significant relationship to SMA type or functional status. Being that the majority of individuals with SMA are non-ambulatory, a question related to individuals being involved in traditional sports, may be deemed non-applicable. This further highlights the need for more SMA-specific outcomes that includes items of most relevance by function.

\section{Discussion}

\section{Overall burden}

The data here presented examined various PROMs that assess specific aspects of the patient experience that might be more sensitive in capturing subtle, but meaningful changes with drug therapy administration and thereby benchmark the progression of SMA, and other aspects of disease impact such as quality of life, work productivity, and fatigue. Leveraging its annual online Community Update Survey [20], in 2019 Cure SMA engaged respondents in completing three commonly used PROMs: the Health Utilities Index (HUI), Work Productivity and Activity Impairment (WPAI) and the Patient Reported Outcomes Measurement Information System Fatigue Short Form (PROMIS Fatigue SF). These instruments were chosen to quantitatively measure various quality of life aspects impacted by SMA as studied qualitatively through previous studies in an SMA population $[7,29,33]$.

Results demonstrate a highly rated burden of SMA across all three assessments that were measured, supporting findings from previous qualitative studies that assessed the burden of SMA across phenotypes [7, 11, 13]. As measured by the HUI, the quality of life scores fell under the category of 'severe disability;' work productivity lost due to having SMA or caring for someone with SMA was also significant; and the fatigue levels of children affected with SMA was greater than that of the general population regardless of type. Nonetheless, given the validation (age range) and parameters (mobility, emotional, etc.) assessed in each scale, we anticipate that different scales may be appropriate for different SMA subtypes and ages. By conducting similar assessments 
Table 4 WPAI Sub-scores Stratified by Caregiver and Affected Individual

\begin{tabular}{|c|c|c|c|c|c|c|c|}
\hline \multirow[t]{2}{*}{ Functional Status } & \multicolumn{2}{|l|}{ Caregiver } & \multicolumn{2}{|c|}{ Affected Adult } & \multicolumn{2}{|l|}{ Overall } & \multirow{2}{*}{$\begin{array}{l}\text { Significance testing } \\
\text { P value }\end{array}$} \\
\hline & Mean (SD) & Sample Size & Mean (SD) & Sample Size & Mean (SD) & Sample Size & \\
\hline \multicolumn{8}{|l|}{ Permanent Ventilation } \\
\hline Absenteeism, \% & $7.6(9.1)$ & 4 & N/A & N/A & $6.1(8.6)$ & 5 & N/A \\
\hline Presenteeism, \% & $76.4(31.1)$ & 11 & N/A & N/A & $73.1(34.7)$ & 13 & N/A \\
\hline Overall Impairment, \% & $59.0(43.3)$ & 4 & N/A & N/A & $49.2(43.4)$ & 5 & N/A \\
\hline Activity Impairment, \% & $83.1(20.9)$ & 35 & $68.8(25.3)$ & 8 & $80.5(22.2)$ & 43 & $0.13^{b}$ \\
\hline \multicolumn{8}{|l|}{ Non-sitters } \\
\hline Absenteeism, \% & $13.3(15.1)$ & 16 & $6.4(13.6)$ & 15 & $10.0(14.6)$ & 31 & $0.09^{\mathrm{b}}$ \\
\hline Presenteeism, \% & $47.0(31.8)$ & 23 & $36.7(29.5)$ & 18 & $42.4(30.9)$ & 41 & 0.29 \\
\hline Overall Impairment, \% & $50.1(27.3)$ & 16 & $37.2(28.8)$ & 15 & $43.9(28.3)$ & 31 & 0.21 \\
\hline Activity Impairment, \% & $60.8(29.4)$ & 50 & $69.1(21.5)$ & 34 & $64.2(26.7)$ & 84 & 0.14 \\
\hline \multicolumn{8}{|l|}{ Sitters } \\
\hline Absenteeism, \% & $18.8(29.0)$ & 36 & $6.8(12.1)$ & 31 & $13.3(23.4)$ & 67 & $0.04^{*}$ \\
\hline Presenteeism, \% & $41.6(31.0)$ & 45 & $44.5(30.1)$ & 40 & $42.9(30.5)$ & 85 & 0.66 \\
\hline Overall Impairment, \% & $44.6(29.6)$ & 33 & $41.0(23.7)$ & 30 & $42.9(26.8)$ & 63 & 0.59 \\
\hline Activity Impairment, $\%$ & $60.9(25.2)$ & 123 & $55.2(22.6)$ & 65 & $58.9(24.4)$ & 188 & $0.09^{b}$ \\
\hline \multicolumn{8}{|l|}{ Walk with Support } \\
\hline Absenteeism, \% & $16.3(11.5)$ & 6 & $8.5(11.8)$ & 4 & $13.2(11.6)$ & 10 & 0.34 \\
\hline Presenteeism, \% & $55.0(31.2)$ & 8 & $50.0(33.7)$ & 4 & $53.3(30.6)$ & 12 & $0.93^{b}$ \\
\hline Overall Impairment, \% & $62.7(19.5)$ & 6 & $53.2(35.5)$ & 4 & $58.9(25.6)$ & 10 & $0.59^{b}$ \\
\hline Activity Impairment, \% & $50.0(26.5)$ & 27 & $7.0(61.4)$ & 7 & $52.4(26.3)$ & 34 & $0.22^{\mathrm{b}}$ \\
\hline \multicolumn{8}{|l|}{ Walk Independently } \\
\hline Absenteeism, \% & $4.4(8.1)$ & 6 & $6.8(15.1)$ & 12 & $6.0(12.9)$ & 18 & $0.91^{\mathrm{b}}$ \\
\hline Presenteeism, \% & $17.5(22.5)$ & 8 & $37.3(25.5)$ & 15 & $30.4(25.8 \%)$ & 23 & 0.07 \\
\hline Overall Impairment, \% & $25.5(26.5)$ & 6 & $35.5(28.1)$ & 12 & $32.2(27.2)$ & 18 & $0.40^{\mathrm{b}}$ \\
\hline Activity Impairment, \% & $41.2(24.2)$ & 25 & $55.0(22.3)$ & 26 & $48.2(24.1)$ & 51 & $0.02^{*, b}$ \\
\hline
\end{tabular}

${ }^{a}$ Significance testing between work productivity of a caregiver and an affected adult

*Statistically significant at $\mathrm{p}<0.05$

${ }^{\mathrm{b}}$ Mann-Whitney test used to test for significance

with these and other measures, we can leverage the results of this study as a baseline for understanding the burden of SMA in our community in the future.

\section{Strengths and limitations of HUI3 for SMA populations}

The results from the HUI3 varied by SMA type and functional status. Unlike the other HUI systems, the HUI3 measures a person's ability to walk and their dexterity which are key manifestations of SMA and vary across subtypes. As shown by the individual HUI attribute scores, there was a statistically significant difference between SMA types when evaluating the scores of ambulation and dexterity. There was also a statistically significant difference between speech attribute scores across the SMA types with type I showing the lowest speech scores representing severe disability. Although future analyses of evaluating HUI attribute scores by SMA type should adjust for age of the affected individual to determine if differences in speech are due to SMA type, age, or a combination of both. Additionally, the HUI3 measures someone's ability to perform daily activities, such as dressing, which is a common activity that patients with SMA would like to improve or maintain $[14,33]$. Assessing the HUI3 before and after treatment would allow the measurement of progression in the SMA community. However, for those who may never gain motor milestones, the HUI3 may not be a good measurement to detect any changes over time. Moreover, not all the attributes assessed by the HUI3 may be relevant for any of the SMA population. For example, the HUI3 assesses quality of life through vision and hearing attributes, which are not relevant to the manifestation of SMA across types, and the high attribute scores as shown in this analysis, demonstrate this.

\section{Strengths and limitations of WPAI for SMA populations}

Unlike the HUI and the PROMIS, the WPAI can measure both the quality of life from the caregiver or the affected adult perspective. Interestingly, there was not a statistically significant difference in the WPAI subscores for caregivers and affected adults in the majority of the scores suggesting that SMA affects the work 
Table 5 Absenteeism by age of affected individual, in months

\begin{tabular}{llr}
\hline & Unstandardized Coefficients & Standardized Coefficients \\
\hline Age During Survey, in months & $-0.03(0.01)^{* * *}$ & $-0.29^{* * *}$ \\
Constant & $19.36(2.90)$ & \\
\hline
\end{tabular}

Observations: 131

${ }^{* * *} p<0.01$

productivity the same for both the caregiver and the affected individual. However, the absenteeism score of the WPAI was influenced by the age of the affected individual with higher absenteeism scores among caregivers for younger affected individuals. Moreover, the WPAI results across functional status and SMA type show that the WPAI is sensitive to show the differences in severity across the SMA population. However, most of the affected adults that completed a survey or had a survey completed on their behalf were attending school either full or part-time. Therefore, the work productivity lost may be underrepresented in the total SMA population. A scale that redefines productivity as a student will be beneficial to assessing the entire picture of productivity lost. Lastly, it is not uncommon for families affected with SMA to hire professionals to assist with providing care to the affected individual. A limitation of this study was the demographics and employment status of the caregiver was not captured. It would be of interest to control for differences among caregivers to help parse out the differences in work productivity lost among families.

\section{Strengths and limitations of PROMIS fatigue for SMA}

The PROMIS Fatigue SF is a short 10 question questionnaire that requires little time to complete and offers a parent proxy version that allows fatigue assessment in children. The results from the Community Update Survey show higher levels of reported fatigue among those with SMA when compared to the general population.
However, when converting the PROMIS Fatigue scores into $\mathrm{T}$-scores, the $\mathrm{T}$-scores among those with SMA were less than one standard deviation above the general population mean. Additional analyses analysis suggested that some of these questions are not appropriate for an SMA population and/or cognitive and/or mental factors may be more relevant over physiological factors. Similarly, a recent study by Dunaway Young, et al. assessed the relationship of perceived fatigue to fatigability, function and quality of life in SMA and found that perceived fatigue did not correlate with fatigability or function [30].

\section{Opportunities for future efforts}

It will become increasingly important to measure qualitatively and quantitatively, other aspects of physical/emotional/psychosocial functioning; including but not limited to quality of life (such as activities of daily living, levels of achieved independence, etc.) and fatigue through patient reported outcomes for ongoing reimbursement of currently approved drugs. As new treatments are approved, the community's expectations from a given therapy, will likely evolve. As such, our ability to effectively capture clinically meaningful changes across current functional abilities and through various outcomes (motor function, respiratory function, HRQL, SMA-specific PROMs, etc.) must also evolve to ensure we are able to effectively capture meaningful change across the SMA community as therapeutic options evolve.

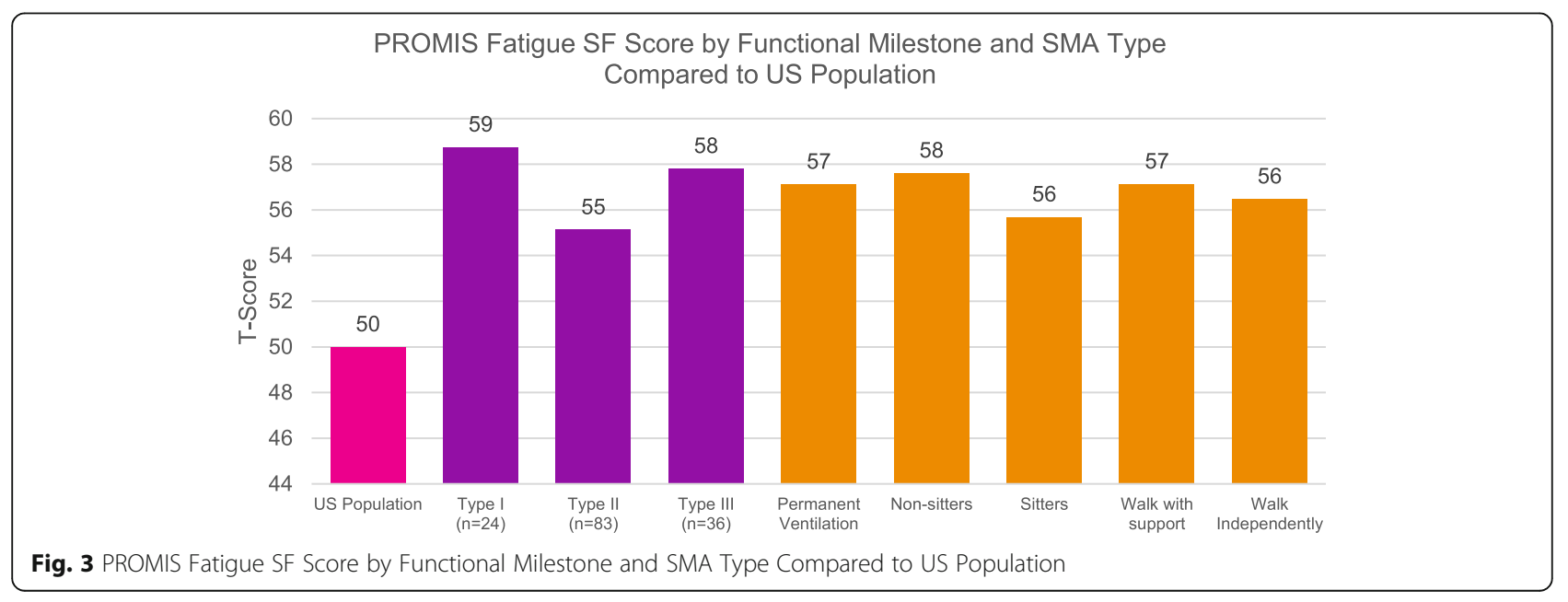


Table 6 PROMIS Fatigue SF T-Scores by Functional Milestone and SMA Type

\begin{tabular}{|c|c|c|c|}
\hline Functional Status & Type I & Type II & Type III \\
\hline \multicolumn{4}{|c|}{ Permanent Ventilation } \\
\hline$n$ & 17 & 2 & 0 \\
\hline Mean (SD) & $56.6(10.2)$ & $62(11.3)$ & \\
\hline Range & $(42-71)$ & $(54-70)$ & \\
\hline \multicolumn{4}{|l|}{ Non-sitters } \\
\hline $\mathrm{n}$ & 2 & 16 & 0 \\
\hline Mean (SD) & $56(8.5)$ & $57.8(10.2)$ & \\
\hline Range & $(50-62)$ & $(39-71)$ & \\
\hline \multicolumn{4}{|l|}{ Sitters } \\
\hline $\mathrm{n}$ & 5 & 55 & 7 \\
\hline Mean (SD) & $67.2(9.5)$ & $54.3(9.0)$ & $58.4(5.4)$ \\
\hline Range & $(54-80)$ & $(34-74)$ & $(48-65)$ \\
\hline \multicolumn{4}{|l|}{ Walk with Support } \\
\hline $\mathrm{n}$ & 0 & 7 & 9 \\
\hline Mean (SD) & & $56.4(8.9)$ & $57.7(7.4)$ \\
\hline Range & & $(39-63)$ & $(47-67)$ \\
\hline \multicolumn{4}{|c|}{ Walk Independently } \\
\hline$n$ & 0 & 3 & 20 \\
\hline Mean (SD) & & $48.7(8.4)$ & $57.7(9.4)$ \\
\hline Range & & $(39-54)$ & (34-73) \\
\hline
\end{tabular}

-Only for unique individuals

\section{Conclusions}

The 2019 Community Update Survey dataset provides an important benchmark from which to begin assessing year-over-year change in HRQoL for affected individuals and their caregivers. Cure SMA will conduct follow up annual surveys using the WPAI and HUI instruments to evaluate the impact that new therapies are making on the overall experience of affected individuals and their families. It is anticipated that these future survey activities will also add in other HRQoL measurements (including the EQ-5D [34] and the Fatigue Impact Scale [35]) to broaden the picture of SMA impact among the community, evaluate which tools are most sensitive to each subtype of the diverse SMA population, can assess treatment affects and determine the health utility among a large sample of affected individuals with SMA.

Ultimately, we anticipate learning through this process, that different instruments will be more appropriate for assessing HRQoL within SMA, and among the various SMA subpopulations, ages of affected individuals and functional milestone status.

\section{Supplementary information}

Supplementary information accompanies this paper at https://doi.org/10. 1186/s13023-020-01498-2.

Additional file 1.

\section{Acknowledgements}

The authors would like to acknowledge all the members of the SMA community who have supported this important survey project. We are extraordinarily grateful to the affected individuals and their caregivers who took the time to respond to the survey. Their willingness to share details of the impact of SMA on their families and daily lives allows us to advance understanding of this disease and lay the foundation for continued progress on behalf of our community.

We are also grateful to Wendy K.D. Selig, of WSCollaborative, LLC, for assisting with the development of this manuscript.

\section{Authors' contributions}

LB collected and analyzed the patient data. LB, RC, and JJ were major contributors of the study design and interpretation of the patient data. All authors contributed to writing the manuscript. All authors read and approved the final manuscript.

\section{Funding}

Funding for this research was supported by the Cure SMA Industry Collaboration. The members of the Cure SMA Industry Collaboration at the time of this study were, AveXis, Inc., Biogen, Genentech/ Roche Pharmaceuticals, Novartis, Cytokinetics Inc., Astellas, and Scholar Rock, Inc.

\section{Availability of data and materials}

The data collected and analyzed during the current study is generated and owned by Cure SMA and not publicly available.

\section{Ethics approval and consent to participate}

All procedures performed in studies involving human participants were in accordance with the ethical standards of the institutional and/or national research committee and with the 1964 Helsinki declaration and its later amendments or comparable ethical standards. Ethics approval for this study was granted by Western IRB (IRB Report ID: 1182230). Informed consent was obtained from all individual participants included in the study.

\section{Consent for publication}

Not applicable.

\section{Competing interests}

The authors have no competing interests to disclose.

Received: 5 May 2020 Accepted: 7 August 2020

Published online: 24 August 2020

\section{References}

1. Arnold WD, Kassar D, Kissel JT. Spinal muscular atrophy: diagnosis and management in a new therapeutic era. Muscle Nerve. 2015;51(2):157-67.

2. Kolb SJ, Kissel JT. Spinal muscular atrophy: a timely review. Arch Neurol. 2011;68(8):979-84.

3. Finkel RS, McDermott MP, Kaufmann P, Darras BT, Chung WK, Sproule DM, et al. Observational study of spinal muscular atrophy type I and implications for clinical trials. Neurology. 2014;83(9):810-7.

4. De Vivo DC, Bertini E, Swoboda K, Hwu W-L, TO C, Finkel RS, et al. Nusinersen initiated in infants during the presymptomatic stage of spinal muscular atrophy: interim efficacy and safety results from the phase 2 NURT URE study. Neuromuscul Disord. 2019;29(11):842-56.

5. Mercuri E, Darras BT, Chiriboga CA, Day JW, Campbell C, Connolly AM, et al. Nusinersen versus sham control in later-onset spinal muscular atrophy. $\mathrm{N}$ Engl J Med. 2018;378(7):625-35.

6. Belter LC, Paradis A, Jhaveri M, Reyna S, Hobby K. Cure SMA membership: findings from the 2018 membership survey [abstract P.94]. Neuromuscular Disord. 2018;28(Suppl. 2):S58.

7. Qian Y, McGraw S, Henne J, Jarecki J, Hobby K, Yeh WS. Understanding the experiences and needs of individuals with spinal muscular atrophy and their parents: a qualitative study. BMC Neurol. 2015;15:217.

8. Zerres $\mathrm{K}$, Rudnik-Schoneborn S. Natural history in proximal spinal muscular atrophy. Clinical analysis of 445 patients and suggestions for a modification of existing classifications. Arch Neurol. 1995;52(5):518-23.

9. Waldrop MA, Kolb SJ. Current treatment options in neurology-SMA therapeutics. Curr Treat Options Neurol. 2019;21(6):25. 
10. Finkel RS, Mercuri E, Darras BT, Connolly AM, Kuntz NL, Kirschner J, et al. Nusinersen versus sham control in infantile-onset spinal muscular atrophy. N Engl J Med. 2017;377(18):1723-32.

11. Wan HWY, Carey KA, D'Silva A, Kasparian NA, Farrar MA. "Getting ready for the adult world": how adults with spinal muscular atrophy perceive and experience healthcare, transition and well-being. Orphanet J Rare Dis. 2019; 14(1):74.

12. Droege M, Sproule D, Arjunji R, Gauthier-Loiselle M, Cloutier M, Dabbous O. Economic burden of spinal muscular atrophy in the United States: a contemporary assessment. J Med Econ. 2020;23(1):70-9.

13. Mongiovi P, Dilek N, Garland C, Hunter M, Kissel JT, Luebbe E, et al. Patient reported impact of symptoms in spinal muscular atrophy (PRISM-SMA). Neurology. 2018;91(13):e1206-e14.

14. Cruz R, Lenz M, Belter L, Hobby K, Jarecki J. The voice of the patient report for spinal muscular atrophy; 2018.

15. Cruz R, Belter L, Wasnock M, Nazarelli A, Jarecki J. Evaluating Benefit-risk Decision-making in Spinal Muscular Atrophy: A First-ever Study to Assess Risk Tolerance in the SMA Patient Community. Clin Ther. 2019;41(5):943-60.e4.

16. Institute for Clinical and Economic Review. Spinraza and Zolgensma for spinal muscular atrophy: effectiveness and value. Final evidence report. 2019. Available at: https://icer-review.org/wp-content/uploads/2018/07/ ICER_SMA_Final_Evidence_Report_052419.pdf. (updated May 24, 2019).

17. Public Comments on ICER report 2019. Available from: https://icer-review. org/wp-content/uploads/2018/07/ICER_SMA_Public_Comments_022219.pdf Accessed 2 Feb 2020

18. Wong W. Should ICER Look at transformative therapies? Journal of clinical pathways; 2019.

19. Landfeldt E, Lindgren P, Bell CF, Guglieri M, Straub V, Lochmuller H, et al. Health-related quality of life in patients with Duchenne muscular dystrophy: a multinational, cross-sectional study. Dev Med Child Neurol. 2016;58(5):508-15.

20. Cure SMA Launches Third Annual Community Survey to Address Important Issues in SMA Treatment: Cure SMA; 2019. Available from: https://www. curesma.org/cure-sma-launches-third-annual-community-survey-to-addressimportant-issues-in-sma-treatment/.

21. Belter L, Cook SF, Crawford TO, Jarecki J, Jones CC, Kissel JT, et al. An overview of the Cure SMA membership database: highlights of key demographic and clinical characteristics of SMA members. J Neuromuscul Dis. 2018;5(2):167-76.

22. Iannaccone ST, Hynan LS, Morton A, Buchanan R, Limbers CA, Varni JW, et al. The PedsQL in pediatric patients with spinal muscular atrophy: feasibility, reliability, and validity of the pediatric quality of life inventory generic Core scales and neuromuscular module. Neuromuscul Disord. 2009; 19(12):805-12

23. Belter L, Jarecki J, Hobby K, Teynor M. Family impact and health-related quality of life (HRQOL) of parents and individuals with SMA. Neuromuscul Disord. 2017;27:S224.

24. Horsman J, Furlong W, Feeny D, Torrance G. The health utilities index (HUI): concepts, measurement properties and applications. Health Qual Life Outcomes. 2003;1:54.

25. Patrick D, Erickson P. Health status and health policy: quality of life in health care evaluation and resource allocation. New York: Oxford University Press; 1993. p. 478.

26. Feng $Y$, Bernier J, McIntosh C, Orpana H. Validation of disability categories derived from health utilities index mark 3 scores. Health Rep. 2009;20(2):43-50.

27. Reilly M, Zbrozek A, Dukes E. The validity and reproducibility of a work productivity and activity impairment instrument. Pharmacoeconomics. 1993;4(5):353-65.

28. Messina S, Frongia AL, Antonaci L, Pera MC, Coratti G, Pane M, et al. A critical review of patient and parent caregiver oriented tools to assess health-related quality of life, activity of daily living and caregiver burden in spinal muscular atrophy. Neuromuscul Disord. 2019;29(12):940-50.

29. McGraw S, Qian Y, Henne J, Jarecki J, Hobby K, Yeh WS. A qualitative study of perceptions of meaningful change in spinal muscular atrophy. BMC Neurol. 2017;17(1):68.

30. Dunaway Young S, Montes J, Kramer SS, Podwika B, Rao AK, De Vivo DC. Perceived fatigue in spinal muscular atrophy: a pilot study. J Neuromuscul Dis. 2019;6(1):109-17.

31. Bartels B, Habets LE, Stam M, Wadman RI, Wijngaarde CA, Schoenmakers MAGC, et al. Assessment of fatigability in patients with spinal muscular atrophy: development and content validity of a set of endurance tests. BMC Neurol. 2019;19(1):21.
32. Fatigue. A brief guide to the PROMIS Fatigue instruments. Patient-Reported Outcomes Measurement Information System 2019.

33. Rouault F, Christie-Brown V, Broekgaarden R, Gusset N, Henderson D, Marczuk $P$, et al. Disease impact on general well-being and therapeutic expectations of European type II and type III spinal muscular atrophy patients. Neuromuscul Disord. 2017;27(5):428-438.

34. Lloyd AJ, Thompson R, Gallop K, Teynor M. Estimation of the quality of life benefits associated with treatment for spinal muscular atrophy. Clinicoecon Outcomes Res. 2019;11:615-22.

35. Fisk JD, Ritvo PG, Ross L, Haase DA, Marrie TJ, Schlech WF. Measuring the functional impact of fatigue: initial validation of the fatigue impact scale. Clin Infect Dis. 1994;18(Suppl 1):S79-83.

\section{Publisher's Note}

Springer Nature remains neutral with regard to jurisdictional claims in published maps and institutional affiliations.
Ready to submit your research? Choose BMC and benefit from:

- fast, convenient online submission

- thorough peer review by experienced researchers in your field

- rapid publication on acceptance

- support for research data, including large and complex data types

- gold Open Access which fosters wider collaboration and increased citations

- maximum visibility for your research: over $100 \mathrm{M}$ website views per year

At BMC, research is always in progress.

Learn more biomedcentral.com/submissions 